\title{
Adenosine A2A/A2B Receptor Antagonist AB928
}

National Cancer Institute

\section{Source}

National Cancer Institute. Adenosine A2A/A2B Receptor Antagonist AB928. NCI

Thesaurus. Code C159564.

An orally bioavailable antagonist of both the immunomodulatory checkpoint molecules adenosine $A 2 A$ receptor (A2AR; ADORA2A) and A2B receptor (A2BR; ADORA2B), with potential immunomodulating and antineoplastic activities. Upon administration, A2AR/A2BR antag onist AB928 competes with tumor-released adenosine for binding to $A 2 A R$ and A2BR expressed on numerous intra-tumoral immune cells, such as dendritic cells (DCs), natural killer (NK) cells, macrophages and T-lymphocytes. The binding of AB928 to A2AR and A2BR inhibits A2AR/A2BR activity and prevents adenosineA2AR/A2BR-mediated signaling. A2AR/A2BR inhibition activates and enhances the proliferation of various immune cells, abrog ates the adenosine-mediated immunosuppression in the tumor microenvironment (TME) and activates the immune system to exert anti-tumor immune responses against cancer cells, which leads to tumor cell killing. A2AR and A2BR, G protein-coupled signaling receptors, are expressed on the cell surfaces of numerous immune cells. Adenosine is often overproduced by tumor cells and plays a key role in immunosuppression and tumor cell proliferation. 